\section{BRAZIULIAN JOURNAL}

OF MEDICAL AND BIOLOGICAL RESHARCH

www.bjournal.com.br
ISSN 0100-879X

Volume 43 (4) 268-380 April 2011

BIOMEDICAL SCIENCES

AND

CLINICAL INVESTIGATION

Braz J Med Biol Res, April 2011, Volume 44(4) 303-310

doi: 10.1590/S0100-879X2011007500026

Co-culture of chondrocytes and bone marrow mesenchymal stem cells in vitro enhances the expression of cartilaginous extracellular matrix components

Qing Chang, Wei-ding Cui and Wei-min Fan

The Brazilian Journal of Medical and Biological Research is partially financed by
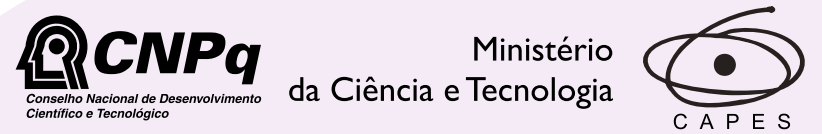

Ministério da Educação
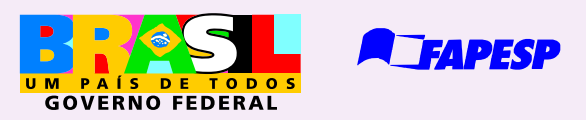

Institutional Sponsors
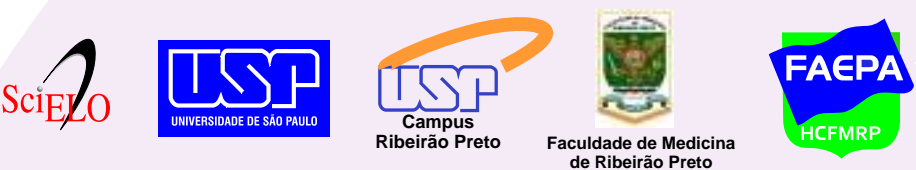

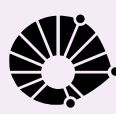

UNICAMP
Ф SHIMADZU

GE Healthcare
Hotsite of proteomics metabolomics developped by:

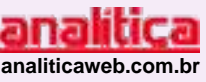




\title{
Co-culture of chondrocytes and bone marrow mesenchymal stem cells in vitro enhances the expression of cartilaginous extracellular matrix components
}

\author{
Qing Chang*, Wei-ding Cui* and Wei-min Fan \\ ${ }^{1}$ Department of Orthopedics, First Affiliated Hospital, Nanjing Medical University, Nanjing, Jiangsu, China
}

\begin{abstract}
Chondrocytes and bone marrow mesenchymal stem cells (BMSCs) are frequently used as seed cells in cartilage tissue engineering. In the present study, we determined if the co-culture of rabbit articular chondrocytes and BMSCs in vitro promotes the expression of cartilaginous extracellular matrix and, if so, what is the optimal ratio of the two cell types. Cultures of rabbit articular chondrocytes and BMSCs were expanded in vitro and then cultured individually or at a chondrocyte:BMSC ratio of 4:1, 2:1, 1:1, 1:2, 1:4 for 21 days and cultured in DMEM/F12. BMSCs were cultured in chondrogenic induction medium. Quantitative real-time RT-PCR and Western blot were used to evaluate gene expression. In the co-cultures, type II collagen and aggrecan expression increased on days 14 and 21 . At the mRNA level, the expression of type II collagen and aggrecan on day 21 was much higher in the 4:1,2:1, and 1:1 groups than in either the articular chondrocyte group or the induced BMSC group, and the best ratio of co-culture groups seems to be $2: 1$. Also on day 21 , the expression of type II collagen and aggrecan proteins in the 2:1 group was much higher than in all other groups. The results demonstrate that the co-culture of rabbit chondrocytes and rabbit BMSCs at defined ratios can promote the expression of cartilaginous extracellular matrix. The optimal cell ratio appears to be 2:1 (chondrocytes:BMSCs). This approach has potential applications in cartilage tissue engineering since it provides a protocol for maintaining and promoting seed-cell differentiation and function.
\end{abstract}

Key words: Co-culture; Bone mesenchymal stem cells; Chondrocytes; Tissue engineering

\section{Introduction}

Chondrocytes and bone marrow mesenchymal stem cells (BMSCs) are frequently used as seed cells in cartilage tissue engineering (1-4). A satisfactory curative effect has been achieved in the clinical application of chondrocytes to the construction of tissue-engineered cartilage and the repair of defects of the articular cartilage (5-7). However, the clinical applications are hindered by the fact that: 1) chondrocytes are highly differentiated cells and their availability is therefore limited and 2) under in vitro conditions they tend to rapidly de-differentiate $(8,9)$. BMSCs have been suggested as a substitute for chondrocytes in cartilage tissue engineering because of their significant proliferative and regenerative capacity $(10,11)$. However, this strategy is problematic because of the low induction efficiency of single BMSC cultures, the potential risk for tumor formation by these cells (12), and the need for growth factors and/or gene delivery systems to signal directed cell growth (13). Thus, novel approaches are needed before BMSCs can be used therapeutically.

The co-culture of stem cells with other mature cells is being increasingly utilized to drive stem cells differentiation toward the needed lineages (14). For example, the microenvironment formed by chondrocytes can promote the differentiation of BMSCs into chondrocytes $(15,16)$ and the cytokines secreted by BMSCs can encourage the proliferation of cartilage cells and the synthesis of cartilaginous extracellular matrix (ECM). Cartilage cells secrete growth factors such as transforming growth factor- $\beta$ (TGF- $\beta$ ) and insulin-like growth factor-1 (IGF-1), which induce chondrocyte differentiation by BMSCs (17). Tsuchiya et al. (18)

Correspondence: Wei-min Fan, Department of Orthopedics, First Affiliated Hospital, Nanjing Medical University, Nanjing, Jiangsu 210029, China. Fax: +86-25-8371-8836-6780. E-mail: fanweimin@vip.sina.com

*These authors contributed equally to this study.

Received October 22, 2010. Accepted February 10, 2011. Available online March 4, 2011. Published April 11, 2011. 
co-cultured human BMSCs and cow chondrocytes and found an increasing rate of propagation of chondrocytes and ECM synthesis.

However, the previous studies have not indicated whether better results could be achieved with the co-culture of chondrocytes and BMSCs than with pure cultures of either chondrocytes or chondrogenically induced BMSCs. Furthermore, the optimal ratio of chondrocytes and BMSCs in co-cultures has not been determined. The purpose of the present study was, firstly, to determine whether coculture of rabbit chondrocytes and BMSCs in vitro promotes the expression of cartilage ECM and, secondly, to define the optimal ratio of the two types of cells by measuring the production of extracellular matrix. Approximately 40$50 \%$ of total cartilage ECM is collagen, with $90 \%$ of total collagen being type II. Proteoglycans, more than $50 \%$ of which are aggrecan, correspond to about $25 \%$ of cartilage ECM. Thus, type II collagen and aggrecan were used to monitor the expression of cartilage ECM.

\section{Material and Methods}

All experimental procedures involving animals conformed with the NIH Guidelines for the Care and Use of Laboratory Animals and were approved by the Administration Committee of Experimental Animals, Jiangsu Province, China.

\section{Isolation, culture, and expansion of bone marrow mesenchymal stem cells}

BMSCs were isolated as described in Ref. 19. Briefly, $6 \mathrm{~mL}$ BMSCs was harvested from the ileum of New Zealand white rabbits $(\mathrm{N}=3)$ and diluted with the same volume of phosphate-buffered saline (PBS) containing $5000 \cup$ heparin. The mixture was separated by density centrifugation through lymphocyte separation solution $(1.073 \mathrm{~g} / \mathrm{mL})$ at $1000 \mathrm{~g}$ for $15 \mathrm{~min}$ at room temperature. The mononuclear fraction interphase was collected and washed twice in PBS. The final pellet was resuspended in $3 \mathrm{~mL}$ Dulbecco's modified Eagle's medium (DMEM)/ F12 (Gibco, USA) supplemented with 10\% fetal bovine serum (FBS; Gibco), $100 \mathrm{U} / \mathrm{mL}$ penicillin $\mathrm{G}$ (Gibco) and $100 \mu \mathrm{g} / \mathrm{mL}$ streptomycin (Gibco), seeded in a $25-\mathrm{cm}^{2}$ culture flask (Corning, USA), and cultured at $37^{\circ} \mathrm{C}$ under a $5 \% \mathrm{CO}_{2}$ atmosphere. The medium was changed every $48 \mathrm{~h}$ and nonadherent hematopoietic cells were removed. After 14 days, the cells had grown to $90 \%$ confluence and were harvested by trypsinization. Two-passaged cells were used. Cell number and viability were assessed with a hemocytometer.

\section{Chondrocyte cultures}

Chondrocytes were isolated from rabbit articular cartilage as described previously (20). In brief, New Zealand white rabbits $(\mathrm{N}=3$ ) weighing about $250 \mathrm{~g}$ were eutha- nized by injection with a Nembutal overdose, and the knee cartilages were removed by sterile dissection. Pieces of cartilage were finely minced and washed with PBS and were then digested with $0.2 \%(\mathrm{w} / \mathrm{v})$ collagenase (Sigma, USA) in PBS for $5 \mathrm{~h}$ at $37^{\circ} \mathrm{C}$. The isolated chondrocytes were resuspended in DMEM/F12 (Gibco) supplemented with $10 \%$ FBS (Gibco), $100 \mathrm{U} / \mathrm{mL}$ penicillin G (Gibco), and $100 \mu \mathrm{g} / \mathrm{mL}$ streptomycin (Gibco). The cells were then plated at a density of $1 \times 10^{5}$ cells $/ \mathrm{mL}$ and placed in a $5 \%$ $\mathrm{CO}_{2}$ incubator at $37^{\circ} \mathrm{C}$. The culture medium was changed every other day.

\section{Evaluation of the potential for multilineage differentiation of BMSCs}

BMSCs at passage 2 were divided into 3 groups: the first group was induced as described by Schilling et al. (21) so that the cells developed into an adipogenic lineage, the second group into an osteogenic lineage (21), and the third group into a chondrogenic lineage (22).

Intracellular lipid vesicles from the adipogenic monolayer cultures were stained with Oil Red O (Sigma) as described by Pittenger et al. (23). Cytoplasmic alkaline phosphatase (ALP) from differentiated or differentiating osteoblasts was stained with ALP solution prepared using a mixture of $80 \mathrm{~mL}$ Naphthol AS-MX phosphate (Sigma) and $2 \mathrm{~mL}$ of fast violet $B$ salt solution (prepared according to manufacturer instructions). The cartilaginous phenotype of the induced cells was confirmed by Alcian blue and Safranin $\mathrm{O}$ staining.

\section{Chondrogenesis of mesenchymal stem cells and chondrocytes}

Chondrocytes and BMSCs of passage 2 were cultured to achieve chondrogenesis. Seven experimental groups were established as follows: in group 1, chondrocytes were cultured in DMEM/F12 (Gibco) supplemented with $10 \%$ FBS (Gibco), $100 \mathrm{U} / \mathrm{mL}$ penicillin G (Gibco), and 100 $\mu \mathrm{g} / \mathrm{mL}$ streptomycin (Gibco); in groups 2-6, chondrocytes and BMSCs were co-cultured at ratios of $4: 1,2: 1,1: 1$, $1: 2$, and $1: 4$ in DMEM/F12 (Gibco) supplemented with $10 \%$ FBS (Gibco), $100 \mathrm{U} / \mathrm{mL}$ penicillin G (Gibco) and 100 $\mu \mathrm{g} / \mathrm{mL}$ streptomycin (Gibco); in group 7, BMSCs were cultured in chondrogenic induction medium (DMEM/F12 supplemented with $10 \%$ FBS, $100 \mathrm{U} / \mathrm{mL}$ penicillin G, 100 $\mu \mathrm{g} / \mathrm{mL}$ streptomycin, $1 \%$ ITS+ (Sigma), $10 \mathrm{ng} / \mathrm{mL}$ TGF- $\beta 1$ (Invitrogen, USA), $0.1 \mu \mathrm{M}$ dexamethasone (Sigma), and 50 $\mu \mathrm{g} / \mathrm{mL}$ ascorbate 2-phosphate (Sigma). In group 8, BMSCs were cultured in DMEM/F12 (Gibco) supplemented with 10\% FBS (Gibco), $100 \mathrm{U} / \mathrm{mL}$ penicillin G (Gibco) and 100 $\mu \mathrm{g} / \mathrm{mL}$ streptomycin (Gibco). Groups 1, 7 and 8 served as the control groups, and groups 2-6 as the experimental groups. All groups initially contained $3 \times 10^{5}$ cells plated at a density of $6 \times 10^{3}$ cells per $\mathrm{cm}^{2}$. The medium was changed every 3 days, and the cells were harvested on days 7, 14, and 21 for analysis. 


\section{Quantitative real-time RT-PCR}

Total RNA was extracted from cell samples using Trizol (Invitrogen). cDNA was synthesized from total RNA using an Omniscript RT kit (Qiagen, USA) according to supplier instructions. Type II collagen and aggrecan mRNA levels were measured by real-time RT-PCR (Stepone real-time PCRApplied Biosystems, USA) using the Fast EvaGreen ${ }^{\circledR}$ master mix for quantitative and high-resolution melting PCR (Biotium, USA). The 20- $\mu \mathrm{L}$ reaction contained 1 $\mu \mathrm{L}$ cDNA from each sample mixed with $10 \mu \mathrm{L} 2 \mathrm{X}$ Fast EvaGreen ${ }^{\circledR}$ qPCR Master Mix, $2 \mu \mathrm{L} 10 \mathrm{X}$ ROX of the assayson-demand kit (Applied Biosystems), $1 \mu \mathrm{L}$ primer, and 6 $\mu \mathrm{L}$ RNase/DNase-free water. The PCR conditions were: incubation at $95^{\circ} \mathrm{C}$ for $2 \mathrm{~min}$ followed by 45 cycles at $95^{\circ} \mathrm{C}$ for $15 \mathrm{~s}$ and at $60^{\circ} \mathrm{C}$ for $60 \mathrm{~s}$. Data were analyzed using the $A B I$ Stepone Sequence Detection Systems software, version 1.0, supplied by Applied Biosystems. The threshold cycle $(\mathrm{Ct})$ value for each sample was defined as the cycle number at which the fluorescence intensity reached a certain threshold at which amplification of each target gene was within the linear region of the reaction amplification curves. The relative expression level for each target gene was normalized by the $\mathrm{Ct}$ value of the housekeeping gene GAPDH using the $2 \Delta \mathrm{Ct}$ relative quantification method, as previously described (24), with the undetected levels treated as zero. In brief, the relative gene copy number was derived using the formula $2-\Delta^{\mathrm{Ct}}$, where $\Delta \mathrm{Ct}$ is the difference in amplification cycles required to detect the amplification products of the target genes (aggrecan and type II collagen) relative to the internal control, GAPDH; namely, relative copy number $=2$-[Ct(target) $-\mathrm{Ct}(\mathrm{GAPDH})]$. Each sample was analyzed in triplicate. PCR was performed using specific primers designed from the published sequence of each cDNA as follows: GAPDH, sense: 5'-CTGCCGCCTGGAGAAAG-3', antisense: 5'-CGACCTGGTCCTCGGTGTA-3'; type II collagen, sense: 5'-GCACCCATGGAC ATTGGAGGG-3', antisense: 5'-GACACG GAGTAGCACCATCG-3'; aggrecan, sense: 5'-GGTCGTGGTGAAAGGTGTTG-3', antisense: 5'-GGTGGAAGCCATCCTCGTA-3'; type I collagen, sense: 5'-AAGAGGAAACTG CAAGAAGGG-3', antisense: 5'-CGTTGGG ACCATCATCACC-3'.

\section{Western blot analysis}

Aliquots of cell lysates containing an equal amount of protein were submitted to SDS-PAGE and transferred onto PVDF membranes (Invitrogen). After blocking in Tris-buffered saline (TBS, pH 7.6) containing $5 \%$ nonfat milk and $0.1 \%$ Tween- 20 , the membranes were probed with type II collagen antibody (CP18, Calbiochem, Germany) and aggrecan antibody (AB-3773, Abcam, USA) at a 1:200 dilution in the blocking buffer and incubated overnight at $4^{\circ} \mathrm{C}$, washed three times with TBST (TBS and $0.1 \%$ Tween-20), incubated with horseradish peroxidase (HRP)-conjugated secondary antibodies (Bio-Rad, USA), and then probed using a SuperSignal West Pico chemiluminescent substrate kit (Pierce, USA). The membranes were scanned using a GS800 Densitometer Scanner (Bio-Rad), followed by data analysis using the PDQuest 7.2.0 software (Bio-Rad).

\section{Statistical analysis}

Data are reported as means $\pm S D$ and were analyzed statistically using the SPSS 10.0 software (LEAD Technologies, Inc., USA). The statistical significance of the differences between groups was determined by one-way analysis of variance (ANOVA). $\mathrm{P}<0.05$ was considered to be statistically significant.

\section{Results}

\section{Analysis of the potential for multilineage differentiation of BMSCs}

To identify the cells isolated from rabbit bone marrow with undifferentiated BMSCs, multilineage differentiations were evaluated. Adipogenesis was confirmed by the presence of neutral lipid vacuoles (lipid droplets) that were strongly positive to Oil Red O staining on day 14 (Figure 1A).
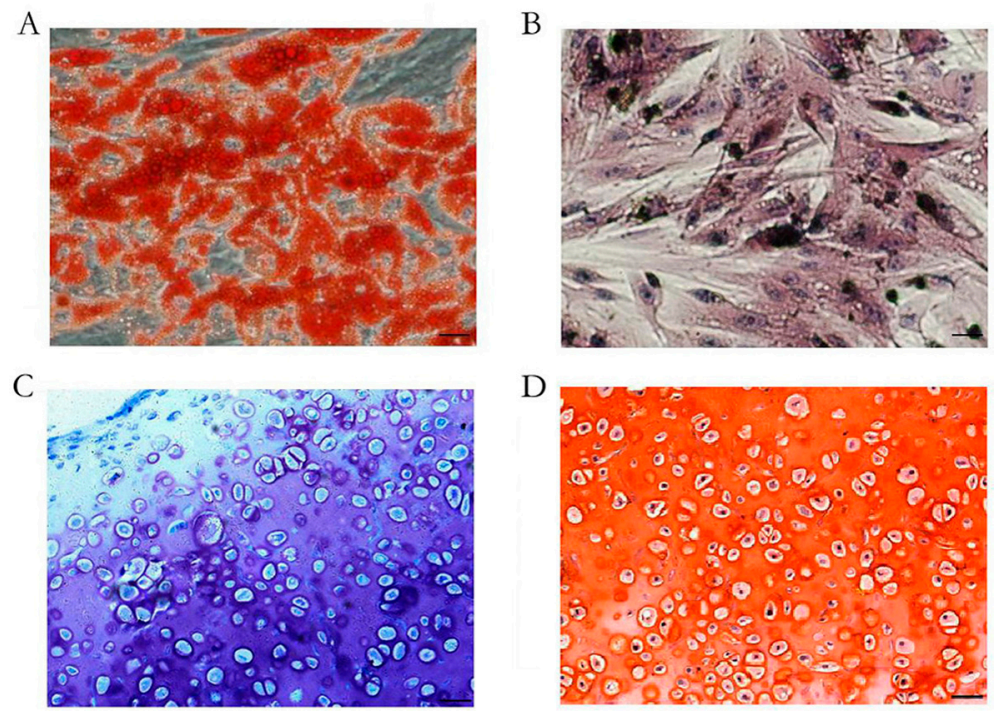

$\mathrm{D}$

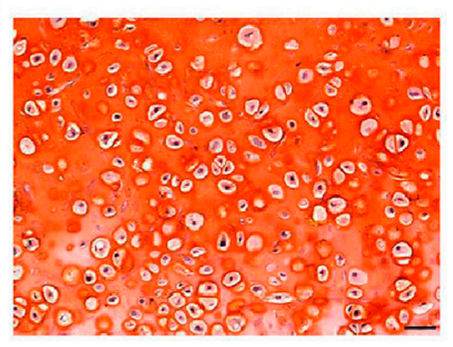

Figure 1. Analysis of the potential for multilineage differentiation of mesenchymal stem cells isolated from rabbit bone marrow. The bone marrow mesenchymal stem cells (BMSCs) were shown to differentiate into mesenchymal lineages. Adipogenesis was confirmed by neutral lipid vacuoles that stained with Oil Red $O$ on day 14 ( $\mathrm{A}$, scale bar $=10 \mu \mathrm{m})$. BMSCs exposed to osteogenic medium for 21 days were strongly positive to alkaline phosphatase staining $(B$, scale bar $=50$ $\mu \mathrm{m})$. Cartilaginous phenotype of the induced cells confirmed by Alcian blue (C, scale bar $=50 \mu \mathrm{m})$ and Safranin O staining $(D$, scale bar $=50 \mu \mathrm{m})$. 
After 21 days of culture, the BMSCs exposed to osteogenic medium were strongly positive to ALP staining (Figure 1B). The cartilaginous phenotype of the induced cells was confirmed by Alcian blue and Safranin O staining (Figure $1 C$ and $D$, respectively).

\section{Quantitative analysis of type II collagen, aggrecan} and type I collagen gene expression

Quantitative real-time RT-PCR of mRNA samples obtained on days 7,14 , and 21 was performed to detect type II collagen gene expression, aggrecan and type I collagen (Figure 2). Expression of the type II collagen gene was lower in all co-culture groups and in the induced BMSC group than in the chondrocyte group on day 7 . On day 14 , the gene expression of type II collagen in the 2:1 group was higher than in the chondrocyte group, whereas expression in the 1:2,1:4, and induced BMSC groups was lower. On day 21, the gene expression of type II collagen was higher in the $4: 1,2: 1,1: 1$, and $1: 2$ groups than in the chondrocyte group, whereas the difference between the $1: 4$ group and the induced BMSC group or the chondrocyte group was not statistically significant. The expression of type II collagen was lower in the BMSC group than in the chondrocyte group on days 7, 14, 21 (Figure 2A). On day 7 , the expression of the aggrecan gene in the $2: 1$ and $1: 1$ groups was higher than in the chondrocyte group but lower than in the other co-culture groups. There was no statistically significant difference between the induced BMSC group and the chondrocyte group. On day 14, the gene expression of aggrecan in the 4:1,2:1, and 1:1 groups was higher than in the chondrocyte group. The differences between the 1:2, 1:4, induced BMSC, and chondrocyte groups were not significant. On day 21, aggrecan gene expression was higher in all co-culture groups and in the induced BMSC group than in the chondrocyte group. The expression of aggrecan in the BMSC group was much lower than in the chondrocyte group on days 7, 14, and 21 (Figure 2B). Expression of type I collagen was lower in all co-culture groups and in the induced BMSC group than in the chondrocyte group on days 7 and 14 . On day 21 , the expression of type I collagen in the $4: 1,1: 1,1: 2$, $1: 4$, and induced BMSC groups was lower in the chondrocyte group, whereas there was no statistically significant difference between the 2:1 group and the chondrocyte group (Figure 2C).

\section{Semi-quantitative analysis of type II collagen and aggrecan protein expression}

Protein samples obtained on days 7,14 , and 21 were subjected to semi-quantitative Western blot analysis to detect the protein expression of type II collagen and aggrecan. There was no statistical difference in type II collagen protein expression in all groups on day 7 . On day 14 , the expression of type II collagen was higher in the 2:1 group than in the chondrocyte group. On day 21 , the expression
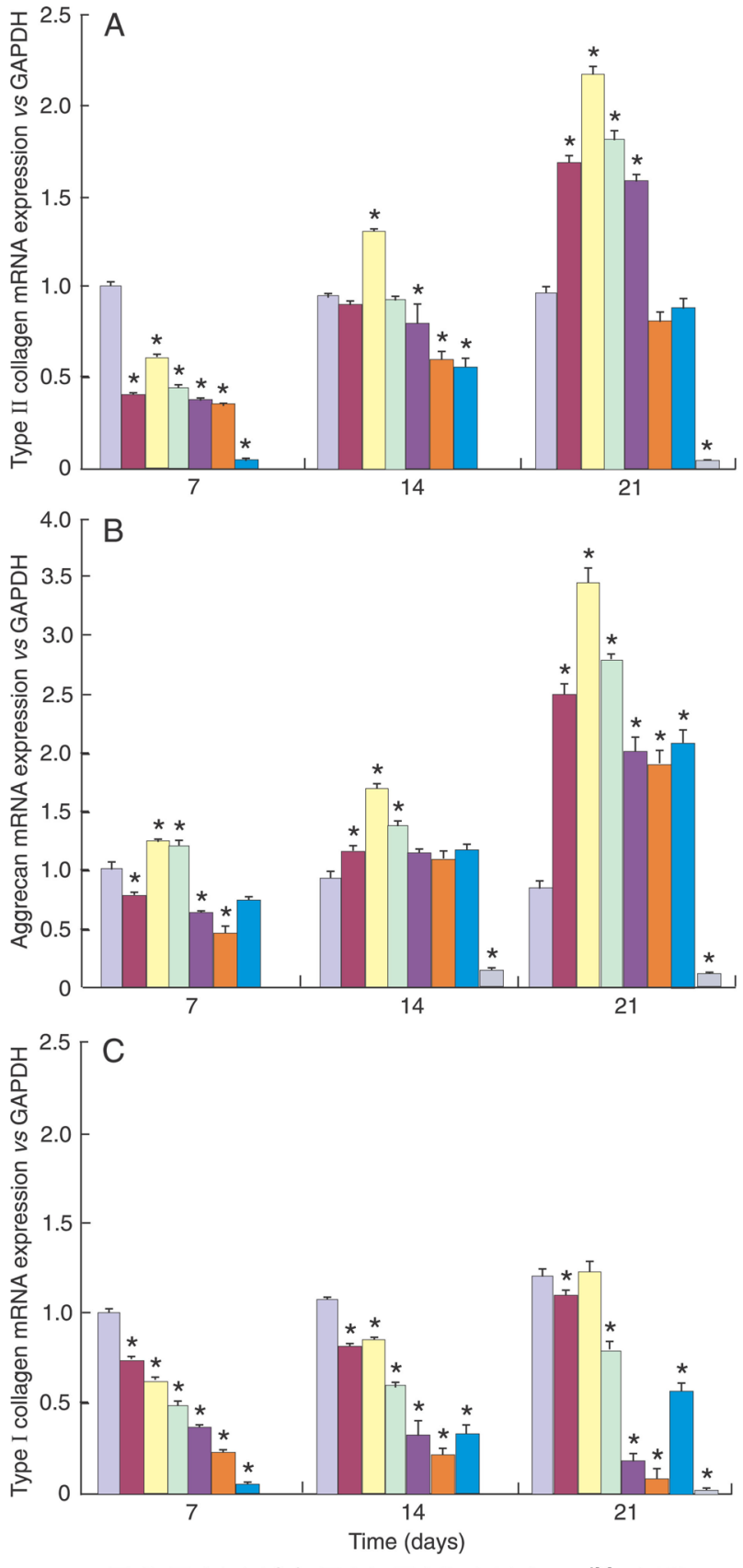

$\square \mathrm{C} \square$ 4:1 $\square 2: 1 \square 1: 1 \square 1: 2 \square 1: 4 \square \mathrm{iM} \square \mathrm{M}$

Figure 2. Quantitative analysis of type II collagen, aggrecan and type I collagen gene expression. The mixed cells at the five ratios of chondrocyte to bone marrow mesenchymal stem cells $(4: 1,2: 1,1: 1,1: 2,1: 4)$ and of the single chondrocyte (C), bone marrow mesenchymal stem cell (M) and induced bone marrow mesenchymal stem cell (iM) groups are shown. Type II collagen, aggrecan and type I collagen mRNA levels were measured by quantitative real-time RT-PCR in the co-culture groups or control groups. Data are reported as means \pm SEM. The experiment was repeated in triplicate for each sample. ${ }^{*} \mathrm{P}<0.05$ vs chondrocyte control culture (one-way ANOVA). 

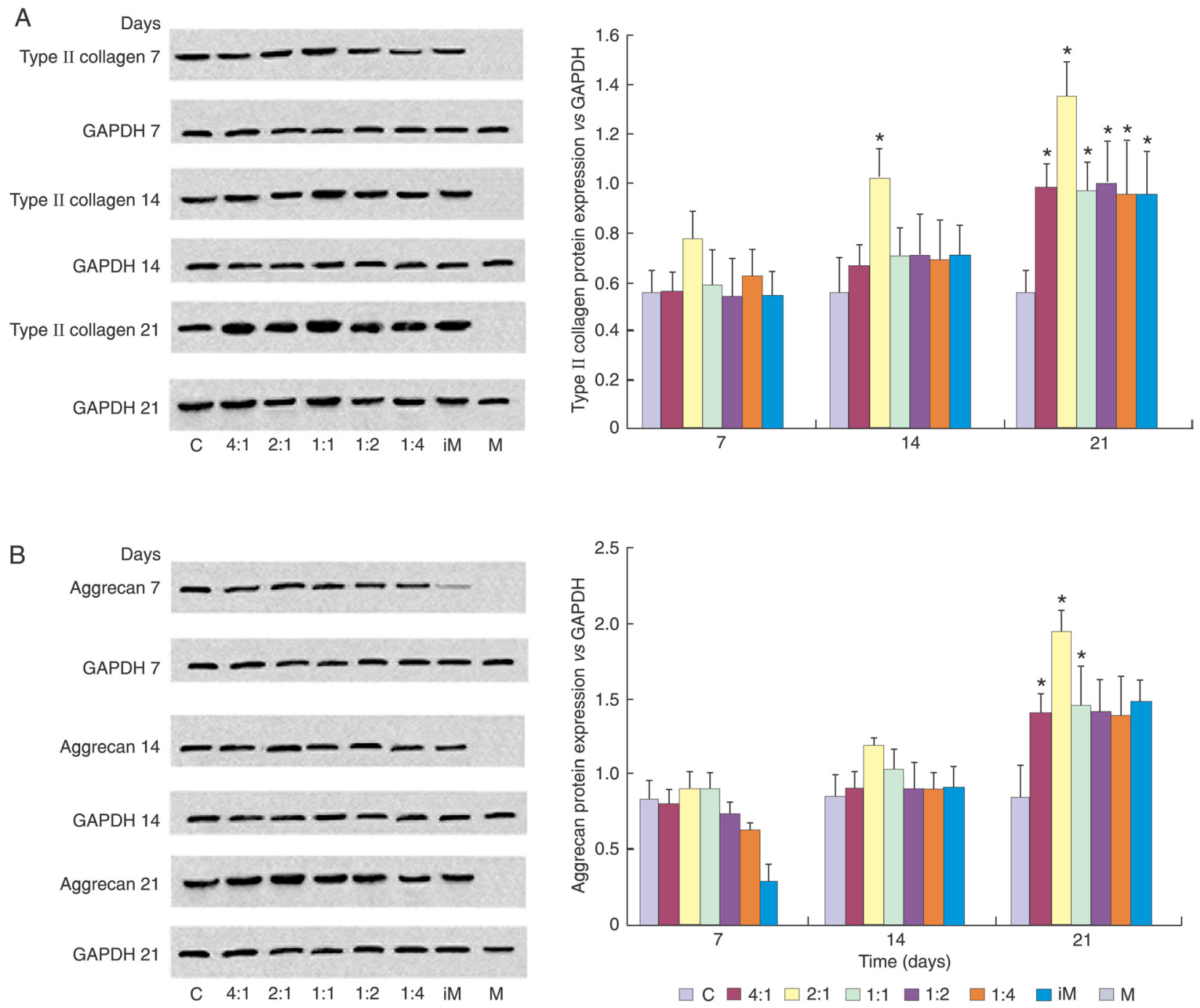

Figure 3. Western analysis of type II collagen and aggrecan protein expression. The mixed cells at the five ratios of chondrocyte to bone marrow mesenchymal stem cell $(4: 1,2: 1,1: 1,1: 2,1: 4)$ and of the single chondrocyte $(C)$, bone marrow mesenchymal stem cell (M) and induced bone marrow mesenchymal stem cell (iM) groups are shown. Type II collagen and aggrecan protein levels were measured by Western blot analysis in co-cultures or control cultures. Representative blots are shown on the left; GAPDH served as the internal control. Data are reported as means \pm SEM. The experiment was repeated in triplicate for each sample. ${ }^{*} P<0.05$ vs chondrocyte control culture (one-way ANOVA).

of type II collagen in all co-culture groups and in the induced BMSC group was higher than in the chondrocyte group (Figure 3A). Aggrecan protein expression in the co-culture groups, the induced BMSC group and the chondrocyte group on days 7 and 14 was not significantly different. On day 21 , the expression of aggrecan in the $4: 1,2: 1$, and 1:1 groups was higher than in the chondrocyte group, whereas there was no statistically significant difference between the $1: 2$, $1: 4$, induced BMSC groups, and the chondrocyte group. The levels of type II collagen and aggrecan in the BMSC group were too low to detect (Figure 3B).

\section{Discussion}

Cell co-culture provides a relatively new approach to overcome the deficits of single culture in vitro. Lin et al. (25) found that the bioactive factors excreted by pancreatic islets can enhance the migration of BMSCs and co-culture can improve islet survival and function. Rat mesenchymal stem cells can be induced to nucleus pulposus-like cells in vitro under the direct influence of intact disc tissue. After 14 days of co-culture, these cells were able to express type II collagen, aggrecan, and sox-9 at the mRNA and 
protein levels (26). Rangappa et al. (27) co-cultured human mesenchymal stem cells and human cardiomyocytes with direct cell-to-cell contact. Their results indicated that human mesenchymal stem cells were plastic and could be reprogrammed into a cardiomyogenic lineage.

To the best of our knowledge, there are only a few reports on the co-culture of chondrocytes and stem cells in cartilage tissue engineering. Human embryonic stem cells co-cultured with articular cartilage cells for 2 weeks significantly increased cartilage matrix production (28). BMSCs co-cultured with human chondrocytes in an inert membrane system increasingly expressed aggrecan and type II collagen after 1 week (29). Mo et al. (30) reported that newly synthesized cartilaginous ECM and type II collagen gene expression were up-regulated after human mesenchymal stem cells (hMSC) and rabbit articular chondrocytes (rAC) were encapsulated together in alginate hydrogels for 28 days. Although the above cited studies found that stem cells could be induced into cartilage cell-like cells and that chondrocytes were supported by stem cells, they did not determine whether the co-culture of chondrocytes and BMSCs was more effective than the single culture of each cell type. The present study showed that type II collagen and aggrecan mRNA levels were higher in the 4:1, 2:1, and 1:1 co-culture groups than in either chondrocyte group or in the induced BMSC group on day 21. Type II collagen and aggrecan protein levels in the 2:1 group were much higher in the chondrocyte group or induced BMSC group on day 21. These findings indicated that the co-culture of rabbit articular chondrocytes and rabbit BMSCs within a defined range of ratios could promote the expression of cartilaginous ECM.

To the best of our knowledge, the study of variations in the ratios of chondrocytes and BMSC co-cultures was only reported by Mo et al. (30). Rabbit articular chondrocytes and human mesenchymal stem cells were co-cultured at different ratios. Their results showed that the GAG and type II collagen content of the 1:2 (rAC:hMSC) group was higher than that of the other co-culture groups or of the BMSC group. However, the difference between the 1:2 (rAC:hMSC) group and the chondrocyte group was not statistically significant. In order to identify the origin of the newly synthesized type II collagen, in their experiment they used the two xenogeneic cell populations. Thus, the optimal ratio of two different cell populations obtained from the same species remains unknown. In the present study, cells from the same species, i.e., rabbit, were mixed at different ratios. On day 21 , the expression of type II collagen and aggrecan at the mRNA and protein levels in the $2: 1$ group were much higher than in all other groups. Thus, the optimal ratio of rabbit chondrocytes and rabbit BMSCs in monolayer co-culture seems to be 2:1 for the expression of the proteins studied.

Type I collagen can be detected in normal articular chondrocytes and was used to measure the osteogenic differentiation of BMSCs. In our study, the expression of type I collagen was reduced as shown by a decreased number of initial chondrocytes on day 7 . The expression of type I collagen in the 4:1 and 2:1 groups was increased with increasing co-culture incubation time, indicating that co-culture generally enhanced gene expression. The expression levels of type II collagen and aggrecan were much higher than that of type I collagen, which is consistent with the results of Richardson et al. (31) who co-cultured human nucleus pulposus cells and human BMSCs (31).

The reason why co-culture of chondrocytes and BMSCs can enhance the expression of cartilaginous ECM is unclear. The microenvironment formed by chondrocytes plays a crucial role in the chondrogeneic differentiation of BMSCs (32). Morphogenetic factors from bovine articular chondrocytes are indispensable in multiple steps during chondrogenic differentiation in vitro and in vivo (33). Chondrogenesis is an orchestrated molecular and cellular process controlled by cellular interactions with growth and morphogenetic factors (34). Variations in the ratio of co-cultured chondrocytes and BMSCs could influence chondrocyte function and chondrogenic differentiation of BMSCs. Moreover, the cellular interactions between chondrocytes and BMSCs in monolayer co-culture are likely to be bi-directional. Chondrocyte-secreted factors such as TGF- $\beta$, bone morphogenetic proteins and IGF-I may promote chondrogenesis of BMSCs in vitro (35-37). Yamamoto et al. (38) co-cultured nucleus pulposus cells with mesenchymal stem cells and found that mesenchymal stem cells can enhance secretion of TGF- $\beta$, IGF- 1 and epidermal growth factors from nucleus pulposus cells. A possible mode of action could be cross talk between cells via gap junctions, which has been observed in coculture of osteoprogenitor cells with endothelial cells where osteogenic differentiation was induced through the gap junction protein connexin 43 (39). These data suggest that chondrocytes can enhance chondrogenesis of BMSCs and at same time BMSCs can improve chondrocyte function in the co-culture system. The neo-chondrocytes will further induce chondrogenic differentiation of BMSCs and ultimately form more chondrocytes.

The 3-D scaffold has been frequently used in cartilage tissue engineering. It is well known that the $3-D$ scaffold closely mimics the environment of articular cartilage in vivo. We suppose that the co-culture of chondrocytes and BMSCs promotes the expression of cartilaginous ECM not only in the monolayer condition but also in the 3-D scaffold. However, the exact results of co-culture of chondrocytes and BMSCs in a 3-D scaffold required further study.

Our study shows that the co-culture of rabbit articular chondrocytes and rabbit BMSCs within a defined range of ratios promotes the expression of cartilaginous extracellular matrix. Under the present conditions described, the optimal ratio seems to be 2:1 (chondrocyte:BMSC). Our method has potential applications in cartilage tissue 
engineering since it provides a protocol for maintaining and promoting seed-cell differentiation and function. However, the optimal ratio of human articular chondrocytes and human BMSCs and the exact mechanisms mediating cell-to-cell interactions between chondrocytes and BMSCs in co-culture require further investigations.

\section{References}

1. Peterson L, Brittberg M, Kiviranta I, Akerlund EL, Lindahl A. Autologous chondrocyte transplantation. Biomechanics and long-term durability. Am J Sports Med 2002; 30: 2-12.

2. Wakitani S, Nawata M, Tensho K, Okabe T, Machida H, Ohgushi $\mathrm{H}$. Repair of articular cartilage defects in the patellofemoral joint with autologous bone marrow mesenchymal cell transplantation: three case reports involving nine defects in five knees. J Tissue Eng Regen Med 2007; 1: 74-79.

3. Kuroda R, Ishida K, Matsumoto T, Akisue T, Fujioka H, Mizuno K, et al. Treatment of a full-thickness articular cartilage defect in the femoral condyle of an athlete with autologous bone-marrow stromal cells. Osteoarthritis Cartilage 2007; 15: 226-231.

4. Rosenberger RE, Gomoll AH, Bryant T, Minas T. Repair of large chondral defects of the knee with autologous chondrocyte implantation in patients 45 years or older. Am J Sports Med 2008; 36: 2336-2344.

5. Scapinelli R, Aglietti P, Baldovin M, Giron F, Teitge R. Biologic resurfacing of the patella: current status. Clin Sports Med 2002; 21: 547-573.

6. Gobbi A, Kon E, Berruto M, Filardo G, Delcogliano M, Boldrini $L$, et al. Patellofemoral full-thickness chondral defects treated with second-generation autologous chondrocyte implantation: results at 5 years' follow-up. Am J Sports Med 2009; 37: 1083-1092.

7. Steinwachs M. New technique for cell-seeded collagenmatrix-supported autologous chondrocyte transplantation. Arthroscopy 2009; 25: 208-211.

8. Marlovits S, Hombauer M, Truppe M, Vecsei V, Schlegel W. Changes in the ratio of type-I and type-II collagen expression during monolayer culture of human chondrocytes. $J$ Bone Joint Surg Br 2004; 86: 286-295.

9. Kang SW, Yoo SP, Kim BS. Effect of chondrocyte passage number on histological aspects of tissue-engineered cartilage. Biomed Mater Eng 2007; 17: 269-276.

10. Sharma B, Elisseeff $\mathrm{JH}$. Engineering structurally organized cartilage and bone tissues. Ann Biomed Eng 2004; 32: 148159.

11. Tuan RS. Stemming cartilage degeneration: adult mesenchymal stem cells as a cell source for articular cartilage tissue engineering. Arthritis Rheum 2006; 54: 3075-3078.

12. Rubio D, Garcia-Castro J, Martin MC, de la Fuente R, Cigudosa JC, Lloyd AC, et al. Spontaneous human adult stem cell transformation. Cancer Res 2005; 65: 3035-3039.

13. Heng BC, Cao T, Lee EH. Directing stem cell differentiation into the chondrogenic lineage in vitro. Stem Cells 2004; 22 : 1152-1167.

14. Sui Y, Clarke T, Khillan JS. Limb bud progenitor cells induce differentiation of pluripotent embryonic stem cells into chondrogenic lineage. Differentiation 2003; 71: 578-585.

\section{Acknowledgments}

Research supported by the National Natural Science Foundation of China (\#30876338 and \#81000800). Jiangsu Key Laboratory of Neuroregeneration of Nantong University is gratefully acknowledged for technical support.

15. Blunk T, Sieminski AL, Gooch KJ, Courter DL, Hollander AP, Nahir AM, et al. Differential effects of growth factors on tissue-engineered cartilage. Tissue Eng 2002; 8: 73-84.

16. Ball SG, Shuttleworth AC, Kielty CM. Direct cell contact influences bone marrow mesenchymal stem cell fate. Int $\mathrm{J}$ Biochem Cell Biol 2004; 36: 714-727.

17. Pedrozo HA, Schwartz Z, Gomez R, Ornoy A, Xin-Sheng W, Dallas SL, et al. Growth plate chondrocytes store latent transforming growth factor (TGF)-beta 1 in their matrix through latent TGF-beta 1 binding protein-1. J Cell Physiol 1998; 177: 343-354.

18. Tsuchiya K, Chen GP, Ushida T, Matsuno T, Tateishi T. The effect of coculture of chondrocytes with mesenchymal stem cells on their cartilaginous phenotype in vitro. Mat Sci Eng C-Bio S 2004; 24: 391-396.

19. Fischer-Rasokat U, Assmus B, Seeger FH, Honold J, Leistner D, Fichtlscherer S, et al. A pilot trial to assess potential effects of selective intracoronary bone marrow-derived progenitor cell infusion in patients with nonischemic dilated cardiomyopathy: final 1-year results of the transplantation of progenitor cells and functional regeneration enhancement pilot trial in patients with nonischemic dilated cardiomyopathy. Circ Heart Fail 2009; 2: 417-423.

20. Ouyang HW, Goh JC, Mo XM, Teoh SH, Lee EH. The efficacy of bone marrow stromal cell-seeded knitted PLGA fiber scaffold for Achilles tendon repair. Ann N Y Acad Sci 2002; 961: 126-129.

21. Schilling T, Noth U, Klein-Hitpass L, Jakob F, Schutze N. Plasticity in adipogenesis and osteogenesis of human mesenchymal stem cells. Mol Cell Endocrinol 2007; 271: 1-17.

22. Johnstone B, Hering TM, Caplan AI, Goldberg VM, Yoo JU. In vitro chondrogenesis of bone marrow-derived mesenchymal progenitor cells. Exp Cell Res 1998; 238: 265-272.

23. Pittenger MF, Mackay AM, Beck SC, Jaiswal RK, Douglas $\mathrm{R}$, Mosca JD, et al. Multilineage potential of adult human mesenchymal stem cells. Science 1999; 284: 143-147.

24. Schmittgen TD, Zakrajsek BA. Effect of experimental treatment on housekeeping gene expression: validation by realtime, quantitative RT-PCR. J Biochem Biophys Methods 2000; 46: 69-81.

25. Lin P, Chen L, Li D, Yang N, Sun Y, Xu Y. Dynamic analysis of bone marrow mesenchymal stem cells migrating to pancreatic islets using coculture microfluidic chips: An accelerated migrating rate and better survival of pancreatic islets were revealed. Neuro Endocrinol Lett 2009; 30: 204-208.

26. Wei A, Chung SA, Tao H, Brisby H, Lin Z, Shen B, et al. Differentiation of rodent bone marrow mesenchymal stem cells into intervertebral disc-like cells following coculture with rat disc tissue. Tissue Eng Part A 2009; 15: 2581-2595.

27. Rangappa S, Entwistle JW, Wechsler AS, Kresh JY. Cardio- 
myocyte-mediated contact programs human mesenchymal stem cells to express cardiogenic phenotype. J Thorac Cardiovasc Surg 2003; 126: 124-132.

28. Bigdeli N, Karlsson C, Strehl R, Concaro S, Hyllner J, Lindahl A. Coculture of human embryonic stem cells and human articular chondrocytes results in significantly altered phenotype and improved chondrogenic differentiation. Stem Cells 2009; 27: 1812-1821.

29. Chen WH, Lai MT, Wu AT, Wu CC, Gelovani JG, Lin CT, et al. In vitro stage-specific chondrogenesis of mesenchymal stem cells committed to chondrocytes. Arthritis Rheum 2009; 60: 450-459.

30. Mo XT, Guo SC, Xie HQ, Deng L, Zhi W, Xiang Z, et al. Variations in the ratios of co-cultured mesenchymal stem cells and chondrocytes regulate the expression of cartilaginous and osseous phenotype in alginate constructs. Bone 2009; 45: 42-51.

31. Richardson SM, Walker RV, Parker S, Rhodes NP, Hunt JA, Freemont AJ, et al. Intervertebral disc cell-mediated mesenchymal stem cell differentiation. Stem Cells 2006; 24: 707-716.

32. Niyibizi C, Wang S, Mi Z, Robbins PD. The fate of mesenchymal stem cells transplanted into immunocompetent neonatal mice: implications for skeletal gene therapy via stem cells. Mol Ther 2004; 9: 955-963.

33. Hwang NS, Varghese S, Puleo C, Zhang Z, Elisseeff J.
Morphogenetic signals from chondrocytes promote chondrogenic and osteogenic differentiation of mesenchymal stem cells. J Cell Physiol 2007; 212: 281-284.

34. Goldring MB, Tsuchimochi K, ljiri K. The control of chondrogenesis. J Cell Biochem 2006; 97: 33-44.

35. Furumatsu T, Tsuda M, Taniguchi N, Tajima Y, Asahara H. Smad3 induces chondrogenesis through the activation of SOX9 via CREB-binding protein/p300 recruitment. J Biol Chem 2005; 280: 8343-8350.

36. Quintana L, zur Nieden NI, Semino CE. Morphogenetic and regulatory mechanisms during developmental chondrogenesis: new paradigms for cartilage tissue engineering. Tissue Eng Part B Rev 2009; 15: 29-41.

37. Zhang M, Zhou Q, Liang QQ, Li CG, Holz JD, Tang D, et al. IGF-1 regulation of type II collagen and MMP-13 expression in rat endplate chondrocytes via distinct signaling pathways. Osteoarthritis Cartilage 2009; 17: 100-106.

38. Yamamoto Y, Mochida J, Sakai D, Nakai T, Nishimura K, Kawada $\mathrm{H}$, et al. Upregulation of the viability of nucleus pulposus cells by bone marrow-derived stromal cells: significance of direct cell-to-cell contact in coculture system. Spine 2004; 29: 1508-1514.

39. Guillotin B, Bourget C, Remy-Zolgadri M, Bareille R, Fernandez P, Conrad V, et al. Human primary endothelial cells stimulate human osteoprogenitor cell differentiation. Cell Physiol Biochem 2004; 14: 325-332. 